\title{
Ondansetron should never be used in pregnancy: AGAINST
}

\author{
Per Damkier ${ }^{1}$, Yusuf Kaplan ${ }^{2}$, Svetlana Shechtman ${ }^{3}$, Orna Diav-Citrin ${ }^{3}$, Matteo Cassina ${ }^{3}$, \\ Corinna Weber-Schoendorfer ${ }^{3}$, Brian Cleary ${ }^{4}$, and Kenneth Hodson ${ }^{3}$ \\ ${ }^{1}$ University of Southern Denmark Department of Clinical Research \\ ${ }^{2}$ Izmir Katip Celebi University \\ ${ }^{3}$ Affiliation not available \\ ${ }^{4}$ The Rotunda Hospital
}

July 16, 2020

In November 2019, section 4.6 of the ondansetron Summary of Product Characteristics (SmPC) was revised by the European Medicines Agency (EMA). It explicitly states, "ondansetron should not be used during the first trimester of pregnancy" (Ondansetron, SmPC[cited 2020 July 08]. Available from: https://www.medicines.org.uk/emc/). Ondansetron is an effective antiemetic drug commonly prescribed as a second-line treatment for severe Nausea and Vomiting of Pregnancy (NVP) (Fiaschi L et al,BJOG 2019;126:1201-11; Oliviera L et al. Obstet Gynecol2014;124:735-42). We do not believe that the underlying evidence supports a strong regulatory discouragement.

Tightening of the SmPC on the use during pregnancy follows EMA assessment of two large pharmacoepidemiological studies, each comprising more than 80,000 first trimester pregnancy exposed liveborn children.

In a methodologically meticulous pharmacoepidemiological study, Huybrechts et al. studied malformation outcomes among 88,000 liveborn children exposed to ondansetron in the first trimester. Comparing to $1,727,000$ unexposed liveborn, they used comprehensive propensity score adjustments for their inferential analysis. Adjusted relative risks were 1.01 (95\% CI 0.98-1.05) for any malformation, 0.99 (95\% CI 0.93-1.06) for cardiac malformations and 1.24 (95\% CI 1.03-1.48) for oral clefts (Huybrechts et al. JAMA 2018;320:2429$37)$.

Subject to substantial academic controversy, Zambelli-Weiner et al. reported data on 82,000 liveborn children exposed to ondansetron in the first trimester (Zambelli-Weiner et al. Reprod Toxicol2019;83:14-20). Overall, they reported a small excess risk of cardiac malformations with an adjusted odds-ratio (aOR) of $1.04(95 \%$ CI 1.00-1.08) and a weak association with orofacial clefts, aOR 1.12 (95\% CI: 0.95-1.33). When restricting exposure to about 5,500 women who were administered ondansetron in a hospital setting, the vast majority of which comprised a single intravenous dose, aOR was 1.43 (95\% CI 1.28-1.61) for cardiac malformations and 1.30 (95\% CI: 0.75-2.25) for orofacial clefts. This study is substantially flawed and we argue it should not be considered when weighing the totality of evidence on the safety of ondansetron in pregnancy due to: 1) strong selection bias 2) poor external validity (the cardiac malformation rate of $3.7 \%$ was extraordinarily high among 780,000 unexposed controls) 3) inadequate adjustment for confounders and covariates and, importantly, 4) serious conflict of interest (Schachtman Law. Re: Zambelli-Weiner [cited 2020 July 8]. Available from: http://schachtmanlaw.com/litigation-science-in-re-zambelli-weiner/ )

In summary, robust clinical data suggest 1) no overall increased risk of major malformations 2) no increased risk of cardiac malformations 3) a small excess risk of oral clefts corresponding to three additional cases of oral cleft for every 10,000 liveborn children exposed to ondansetron in the first trimester of pregnancy (figure $1)$. 
NVP causes significant physical and psychological morbidity to the degree that some women contemplate termination of pregnancy. Denying such patients effective and low-risk treatment is inappropriate. We are confident that women suffering from severe NVP can comprehend the small excess risk of oral clefts in making an informed decision. The statement "ondansetron should never be used in pregnancy" is: 1) misconceived 2) insufficiently substantiated by evidence and 3) not in the best interest of pregnant women.

\section{Disclosure of interests}

The authors declare no conflicts of interest.

\section{Contribution of authorship}

PD conceptualized and drafted the first version of the manuscript. YCK, SS, ODC, MC CWS, BC and KH made critical revisions for important intellectual content and approved the manuscript. BC conceptualized and constructed the figure. PD is the guarantor.

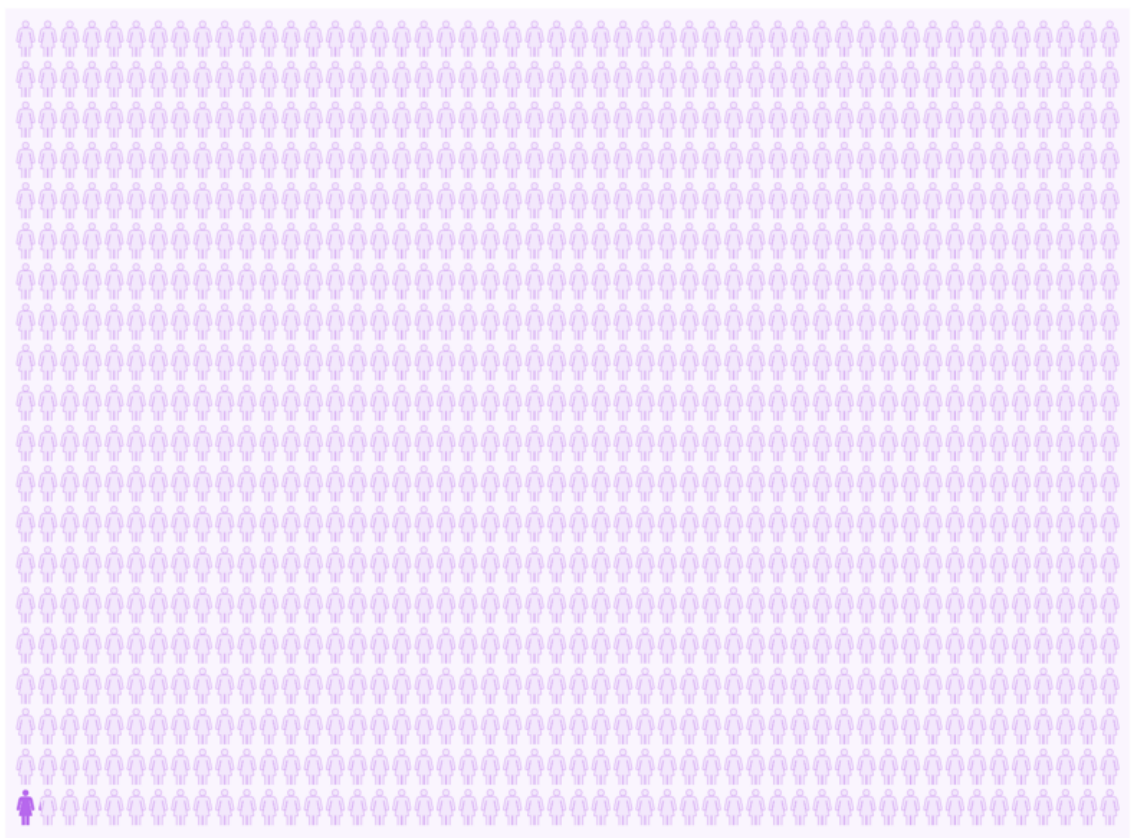




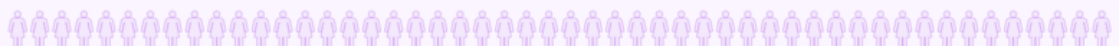

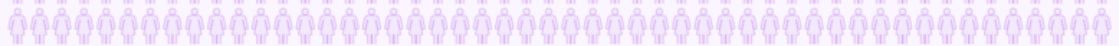

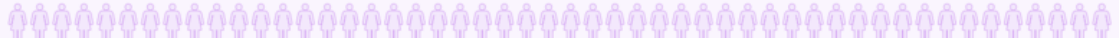

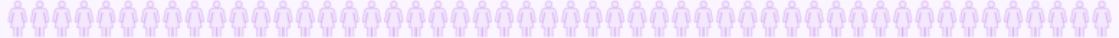

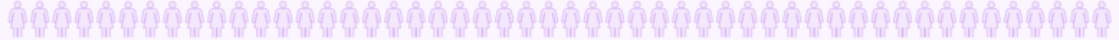
คด

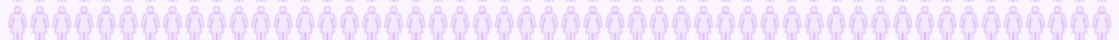

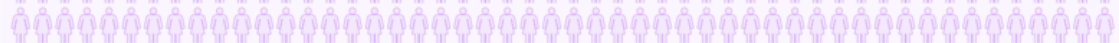

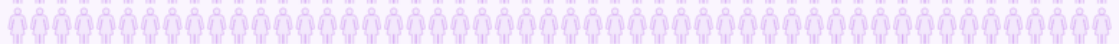

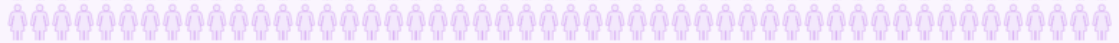

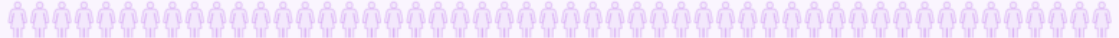

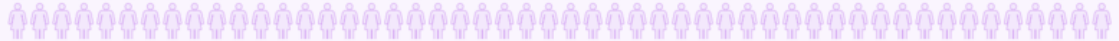

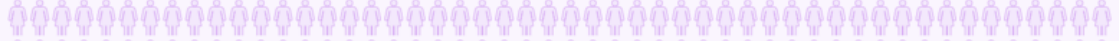

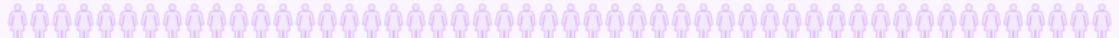

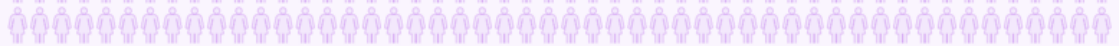

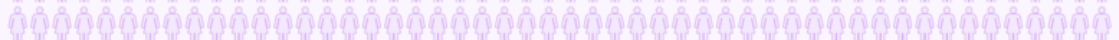

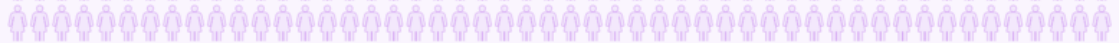

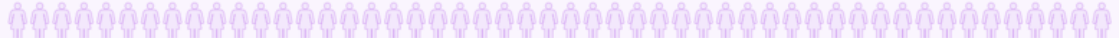

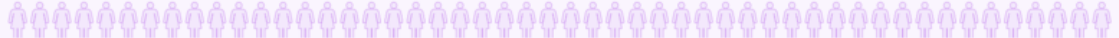
พ 